\title{
HYBRID OPTICAL AMPLIFIER FOR DENSE WAVELENGTH DIVISION MULTIPLEXED SYSTEM
}

\author{
Bhavika Soni, Aabhas Mathur \\ E-Mail Id: sonibhavika75@gmail.com ${ }^{1}$, aabhas08mathur@gmail.com ${ }^{2}$
}

Department of Electronics \& Comm. Engineering, Aravali Institute of Technical Studies, Udaipur, India

Abstract-The fibre system are the important part of modern days communication network. The high rate given by fibre optics can truly be used by employment of WDM techniques. The WDM system is able to cater all value added services (VAS). WDM system long widely accepted in large distant lines suffers from problem such as dispersion and attenuation which results low performance. Dispersion compensation and optical power improvement are necessary parameter in WDM. In the previous work a single FBG is used for compensate the dispersion in single channel and 16 cascaded FBG for 16 channel optical system. In my work EDFA system used to improve power and reduce dispersion and regain higher SNR as compared to single EDFA of some source power. It is also imperative that by placement of stager of EDFA pre and post FBG give instruction for an order to magnitude improvement is SNR and receive excellent dispersion compensation in WDM system.

Keywords: Optical fibre, wavelength division multiplexing (WDM) system, Fibre Bragg grating (FBG), EDFA.

\section{INTRODUCTION}

\subsection{Optical Fibre}

Currently used technique to transfer data in form of light waves. In this technique data transfer speed is high compare to other techniques. [1,3]

\subsection{Wavelength Division Multiplexing}

WDM is a technique which multiplex a number of optical data signals onto single optical fibre at different wavelength.

\subsection{Dispersion compensation fibre}

Electronic balancing strategies are used in the art. Since there is instant recognition in the receiver, direct abortion in the optical space, e.g. Chromatic propagation is converted to non-correct mutations after optical-electrical transformation. It is for this reason that its idea non-Linear Channel displays updated. For this, the structure of front equalizer (FFF) and criticism of choice (DFE) are mainly used. EDC appears at the speed of correspondence as it leads to analogized computerized interactions.[1,2,3,4]

\subsection{Fibre Bragg Grating (FBG)}

Bragg fibre matrices are made by exposing the core of a single-mode fibre laterally to a periodic pattern of intense laser light. The exposure produces a permanent increase in the refractive index of the fibre core, creating a fixed index modulation according to the exposure model. This fixed index modulation is called a network. Each time the refraction changes, a small amount of light are reflected. All reflected light signals combine consistently with a large reflection at a particular wavelength when the cross linking period is about half the wavelength of the incoming light. This is called the Bragg condition and the wavelength at which this reflection occurs is called the Bragg wavelength. Light signals at wavelengths other than Bragg wavelength, which are not adapted by phase, are essentially transparent.

\subsection{Erbium Doped Fibre Amplifier (EDFA)}

An EDFA is device which is used to improve power gain in optical data transfer system. EDFA is now most commonly used to compensate for the loss of optical fibre in long-distance optical communication. Another important feature is that EDFA can amplify multiple optical signals simultaneously and can therefore be easily coupled to WDM technology. $[5,6,7]$

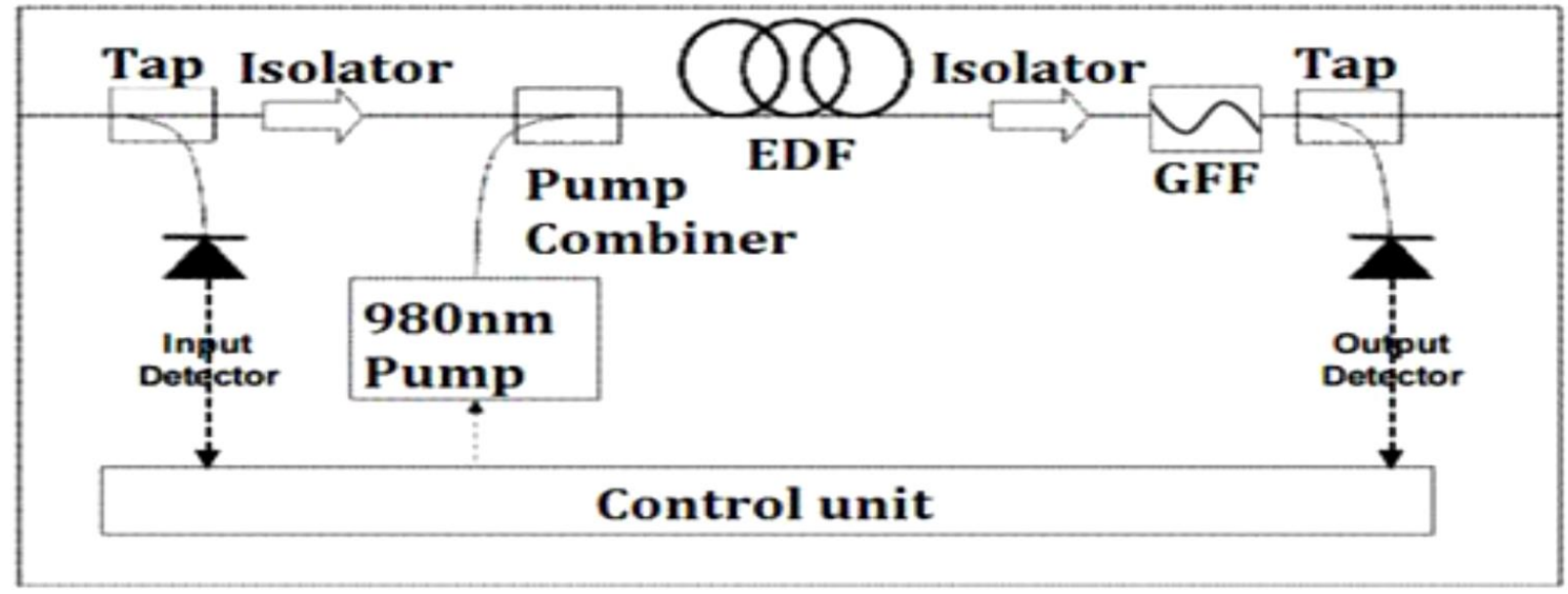

Fig. 2.1 The EDFA setup with $980 \mathrm{~nm}$ pump vitality 


\subsection{Advantages of EDFA}

$>$ EDFA amplifies optical signals without optoelectronic and electrophonic conversion.

$>$ EDFA amplifies multiple optical signals simultaneously.

$>$ The amplification of the optical signal is independent of the data rate.

$>$ High profit

$>$ Low noise data

$>$ It does not require high-speed electronics.

$>$ No high speed deformation.

\section{OBJECTIVE}

$>$ Design \& implementation of an advanced dispersion compensation system for high bandwidth communication links.

$>$ Use of FBG or hybrid grating schemes for optical medium Dispersion compensation.

$>$ Application of EDFA for optical amplification of received signal to overcome fiber/media loss.

$>$ Analysis of Dispersion compensation fiber (DCF) as optical medium to achieve lower dispersion \& higher bandwidth.

$>$ Use of electron dispersion control methods such as digital filters to achieve low dispersion levels.

$>\quad$ Use of hybrid optical amplifier to compensate \& achieve high signal to noise ratio (SNR).

$>\quad$ Use of DSP (digital signal processing) based hardware or software system to dynamically detection \& update Bit stream to achieve low BER (Bit error rate).

$>$ Complete hybrid system using optical \& electronic filters fibers \& amplifier to achieve low power loss \& low dispersion for implementing a true high bandwidth long distance WDM system.

\section{LITERATURE SURVEY}

This article focuses on building a four-channel wavelength division multiplex (WDM) using SOA (semiconductor Optical amplifier) at a speed of $10 \mathrm{Gbit} / \mathrm{s}$ and a dispersion of $1 \mathrm{~nm}$ using a real optical system under specified conditions . The optical line quality for a separate channel is evaluated on the basis of BER (bit blunder rate). The article's side effect is the WDM framework in a mock domain that evaluates BER against a particular SOA flow. The expected wavelengths for traction current in SOA were $1549 \mathrm{~nm}$ and $1550 \mathrm{~nm}: 0.05 \mathrm{~A}, 0.1 \mathrm{~A}$ and $0.2 \mathrm{~A}$. The actual idea of wavelength division in the optical equivalent was therefore described hypothetically and in the remaining $50 . \%$ Represented.[1] However, practical applications followed several years later, largely due to the lack of innovation required for the meeting. The transmission of two wavelengths into a fiber at the main research center was performed efficiently in 1978, and WDM was seriously built and improved for viable application at the time. Currently, WDM frame transmission is a distinctive element of innovation, and an optical fiber transmits dozens or above to multiple optical characters separated by different wavelengths.[2] The entire WDM series consists of n optical sources and localized, with the transmitter controlling the transmitted flag for each wavelength used. The purpose of this article was to find the ideal direct current in SOA in the WDM framework in light of BER for single channel. The negligible part interference frequency required for a line for optical matching frames is 10 to 12.[3] Upon reproducing with a SOA design current of $0.05 \mathrm{~A}$, it was found that the BER estimate for the RX-2 and RX-3 collectors was missing as the BER was around 10-5. At an absorption current of $0.1 \mathrm{~A}$, the BER of the RX-2 receiver was found to be satisfactory for use in optical coupling; His rating is 10-12. The BER was reduced to 10-4 by increasing the SOA current to $0.2 \mathrm{~A}$. As can be seen from hypothetical information and recess tests, it was usable to use an absorption current of $0.1 \mathrm{~N}$ at SOA for the four-channel WDM structure with $1 \mathrm{~nm}$ space in the C-band. [4]

Interestingly, we are currently showing an indirect transmission of 16 channels WDM-PON with 10 Gbit / s up to 60 $\mathrm{km}$. An optical spending scheme of more than $30 \mathrm{~dB}$ was obtained. It relies on self-determined RSOAs and multi-step optimization RF signals. We showed a self-built RSOA response to WDM engineering, in which multi-phase adjustments via the FDM flag were associated with an RSOA with low transmission capability.[5] We have achieved a bidirectional $10 \mathrm{~Gb} / \mathrm{s}$ transmission over $60 \mathrm{~km}$ with an optical spending scheme of $30 \mathrm{~dB}$. The combination of FDM and WDM in FWDM-PON regards a self-seeded system that guarantees an intelligent and adaptable device for highspeed transmission for next-generation optical transmission systems. The basic steps involved in how to mediate the use of optical fiber include: The optical flag is constructed using a transmitter, the flag is transmitted along the fiber, the flag is guaranteed not to be overly distorted or impotent, which you get a flag on optics and convert it into an electric flag. Chromatic scattering occurring in optical fibers is a miracle caused by different wavelengths based on a collection of refractive records that cause an expansion of the impulse to propagate in the OFC.[6] Despite the fact that EDFA (erbium-doped fiber amplifiers) pay for transmission problems, color dispersion using EDFA is not reimbursed. One of the suitable and important components of optical matching structure is fiber optic lattice (FBG). Uniform FBGs are considered as dispersion compensators in each optical match frame. The test system used is the programming of OPTISYSTEM 7.0 reproduction. Each innovation is implemented in OPTISYSTEM 7.0 with 10 Gbit / s and $210 \mathrm{~km}$ transmission fiber. The newly constructed transmission structure is divided taking into account various parameters such as BER, Q calculation, output control, amplification, noise figure and eye level.[7]

In this article we have mimicked an optical transmission framework. If we saw the scatter, we decided to repay it for this reason; we have used and reproduced FBG uniforms. The framework was examined with and without FBG capability. We have found that a similar FBG provides better results for 10 Gbit / s optical energy yield. For an optical matching structure with long separation, dispersion in optical fiber limits the design.[8] The dispersion is controlled using fiber shred. The use of Bragg fiber grinding improves bit error rate and Q calculation. We can conclude that 
uniformly grinding the fiber Bragg gives a better Q variable and a smaller error rate than FBG. In the future, it can be used for varying optical correspondence over a long period of time with high information rates and low pitch. [9] A long-range heartbeat radio reduced the design of fiber-optic radios to light self-stage tweaks for duplex correspondence, with radio units (RAUs) reaching them. A duplex design that works at a rate of 1 bit GHz per second has been verified and weighted based on performance parameters. The most extreme tasks are specialists that transmit information from the base station (BS) to the radio to access the versatile base station on the SMF-28, $40 \mathrm{~km}$ from the main office (CO), and to devices within 300 meters. Work is underway to meet the needs of fast-moving future correspondents who provide multiple RAUs with food delays.[10] The effectiveness of reducing the amplitude of the beat to distribute the remuneration of the fiber is investigated, and the proposed technique in fiber grating. A remarkable standard for intelligent optical semiconductor adapters is being introduced in administration for the speed of information and disseminating expert panels. Intelligent semiconductor optical amplifiers (RSOA) are used by upstream radios to reach units and limit the cost of the ROF framework. Bidirectional radio showed fiber transmission of signals in the age of optical carriers based on the balance of rational rhythm levels. In the duplex RoF framework, signals were transmitted at a total rate of 1 Gbit / s.[11] This work focuses on the practical way of managing compositions and moving movements in BROF design. We have proposed a useful and clever scheme combining the adjustment of autonomous internships based on non-linearity to share usage and offer a sustainable framework. An important corruption of ROF correspondence, which limits the achievement of delayed isolation transfer, is due to the expansion of the heartbeat and impedance of the image. The effectiveness of reducing the amplitude of the beat to distribute the remuneration of the fiber is investigated, and the proposed technique in fiber grating.[12] The 50 km framework has been shown to operate with high quality when attached to a DCF framework. Correlation showed that FBG is used for PWR, but DCF outperformed FBG. With the disclosure of optical fibers, a generalized disturbance occurred in the field of correspondence. Fiber optic correspondence provides fast information, security, adaptability, high data transfer and so on. Variables, such as b. Scattering, weakening, loss, etc., but usually impede execution.[13] Furthermore, it is scattering that fiber affects the rapid transmission of information. Scattering fiber spreads along the fiber and forms a barrier. There are various strategies for the spread of salary. This test is performed to uncover the effects of scattering and to find out how it can be overcome with FBG. Fiber Bragg grating is one of the broad advances to offset the equivalent propagation of optical fibers, and here we have used FBG to compensate for propagation in $80 \mathrm{~Gb} / \mathrm{s}$ WDM with an NRB modulator. Is used on the transmitter side, and in this way the result is thought out. The estimation of the Q component, eye level and bit error rate (BER) is solved for each last channel and its estimation is evaluated. In connection with the test, transmission panels were planned, including laser light as the source, modulator, single mode optical fiber such as channels, fiber Bragg rectification (FBG) dispersion compensator, and optical transmission. [14]

Economical Fiber Innovation (DCF), which is used more frequently, is being considered. Three schemes (precompensation, post-compensation, mixed compensation strategy for 40 Gbit / s without a standard connection and scatter return to zero connections, which reimburse via FBG compensator to update higher information Is performed Optical transmission speed. The goal is to construct quality variables, fiber length, and improved learning in eye charts using explicit adjustment procedures. [15]

\section{METHODOLOGY}

Proposed System Architecture and Implementation:

The WDM Framework uses a programming optisystem to underline EDFA. The structure consists of 16 information signals (channels), perfect multiplexers, 4 isolators, pump lasers; erbium-doped fibres with a length of $5 \mathrm{~m}$, demuliplexors, photo detector pins, and low pass short filters, as shown in the figure. The framework is a balanced wavelength division multiplex flag with a direct separation of $100 \mathrm{GHz}$ in the $193.1 \mathrm{THz}$ wavelength field scheme. The energy of each channel is $0 \mathrm{dBm} .980 \mathrm{~nm}$ pumps are used to bring the doping Iotas to a higher standard of living than $0.75 \mathrm{~W}$. There is an erbium-doped fibre behind each insulator. The first (isolator_1) designed by EDF, pump iteration of $980 \mathrm{~nm}$ laser, energy of $0.75 \mathrm{~W}$, the second insulator designed by EDF (Fiber_1 Doped with Erbium) $5 \mathrm{~m}$ in iteration of $980 \mathrm{~m}$ and the length of laser pump 1 Further power and $0.75 \mathrm{~W}$.

In addition, the scaffold also has a repetition of 193.1 Thz FBG (Bragg Fibre Rings). A third EDF (Perfect Isolator 3) was taken from a third EDF (Erbium Doped Fibre 2) with a length of $5 \mathrm{~m}$ and a third laser of pump 2 with an energy of $980 \mathrm{~nm}$ and an energy of $0.75 \mathrm{~W}$. So, compared to the latter, the right fourth isolator 2 will be followed by an optical fibre 10 consecutive kilometres long.

Subsequently, the two-pin $1 * 2$ dmuliplexer_ 1 of the photo detector for the pins of the photo detectors and the pins of the photo detectors 2 and each pin of the photo detectors with based low-pass filters also detected in the framework.

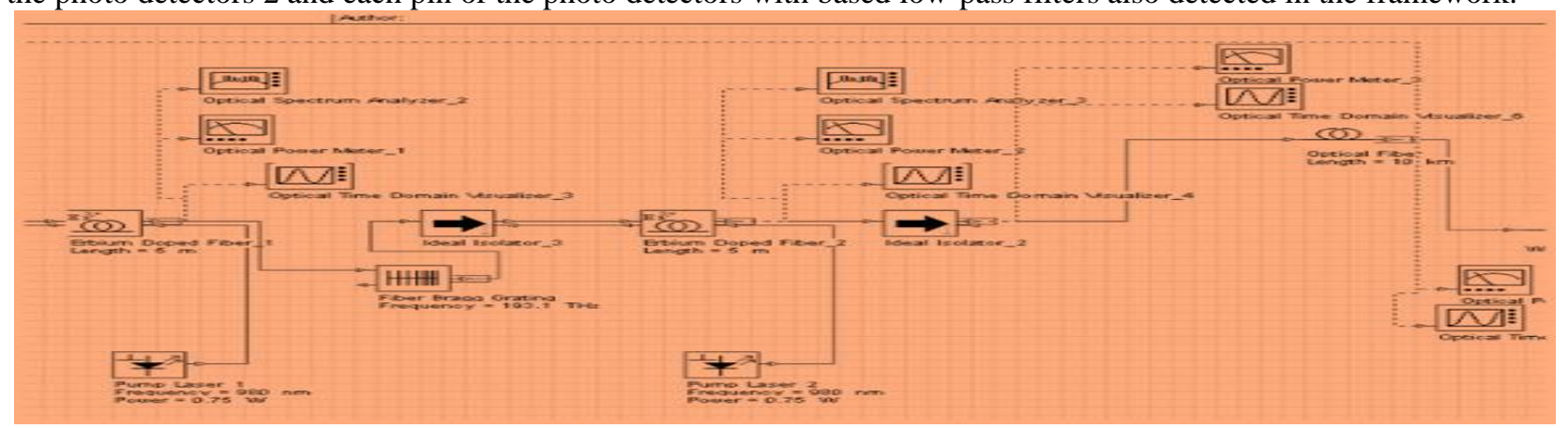

Fig. 5.1 Proposed System Architecture and Implementation

DOI Number: https://doi.org/10.30780/specialissue-SCRDSI-2021/007

Paper Id: IJTRS-SCRDSI-21-007

pg. 26

@ 2017, IJTRS All Right Reserved, www.ijtrs.com 


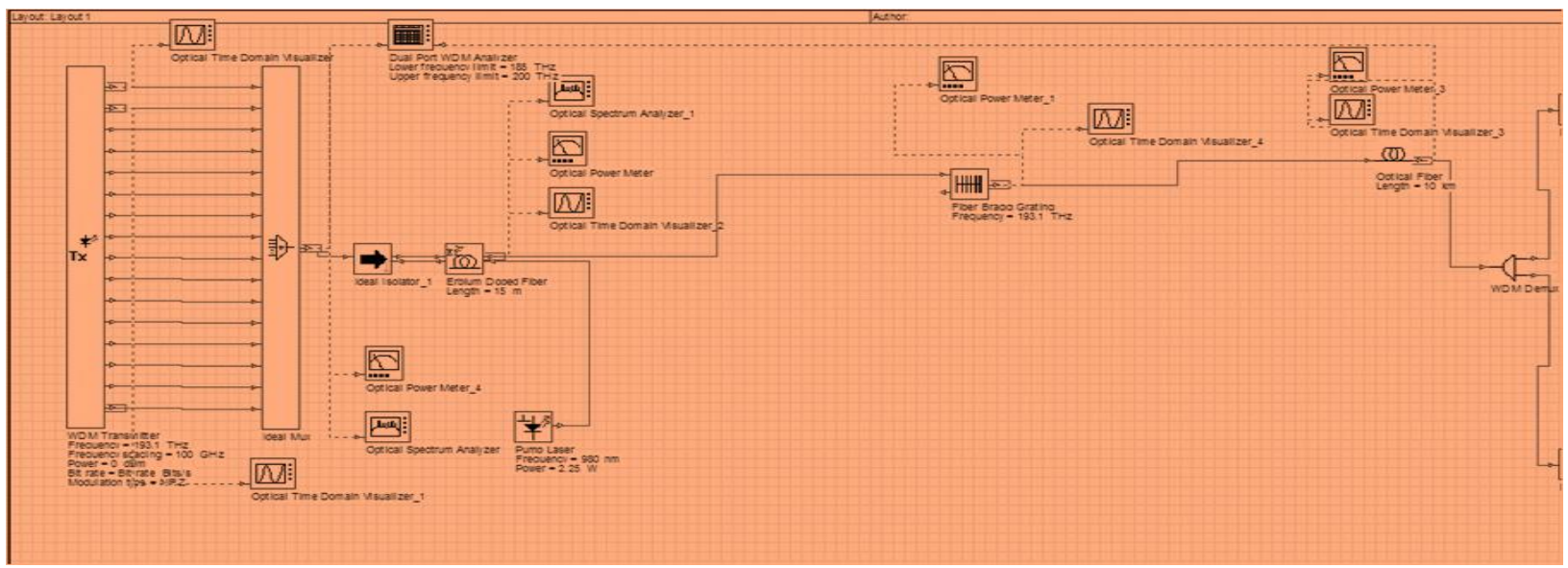

Fig. 5.2 Proposed System Architecture and Implementation

\section{RESULT}

\subsection{Single Stage EDFA}

In this work that we need to depict that contrasts between single stage EDFA and multistage EDFA for optical fibre enhancement. What's more, need to demonstrate that why multistage EDFA is superior to single stage EDFA. In an optical fibre correspondence framework optical signs are transmitted through the optical fibre. At the point when transmitted over long separations, the optical flag is exceedingly mutilated. To anticipate flag debasement over long separation correspondence optical intensifiers are utilized. EDFA is utilized as an optical enhancer in correspondence for better execution. To enhance the execution of the speaker arrange multi pass and multi organize EDFA designs are utilized.

With the Optisystem product, EDFA is described in the WDM Framework. The structure has 16 information signals (channels), a perfect multiplexer, isolators, a pump laser, a erbium-doped fiber with a length of $15 \mathrm{~m}$, de-multiplier, pin for photo search, basal channel. The framework's contribution is the 14 uniform wavelength division multiplexing movements in the 193.1st wavelength localization with the spread of $100 \mathrm{GHz}$ channels. The energy of each channel is $0 \mathrm{dBm}$. Pumping at $980 \mathrm{~nm}$ brings the doped molecules to a higher standard of living with $0.75 \mathrm{~W}$.Withsingle-phase EDFA, when data signals pass throughthe correct multiplexer and data signals which pass through the correct multiplexer, the desired value is first read on the optical power meter (optical power meter_4). Figure 3 shows an estimate of the signal at the beginning of this operation.It is the main measure of knowledge of the estimate of the transmitted flag.

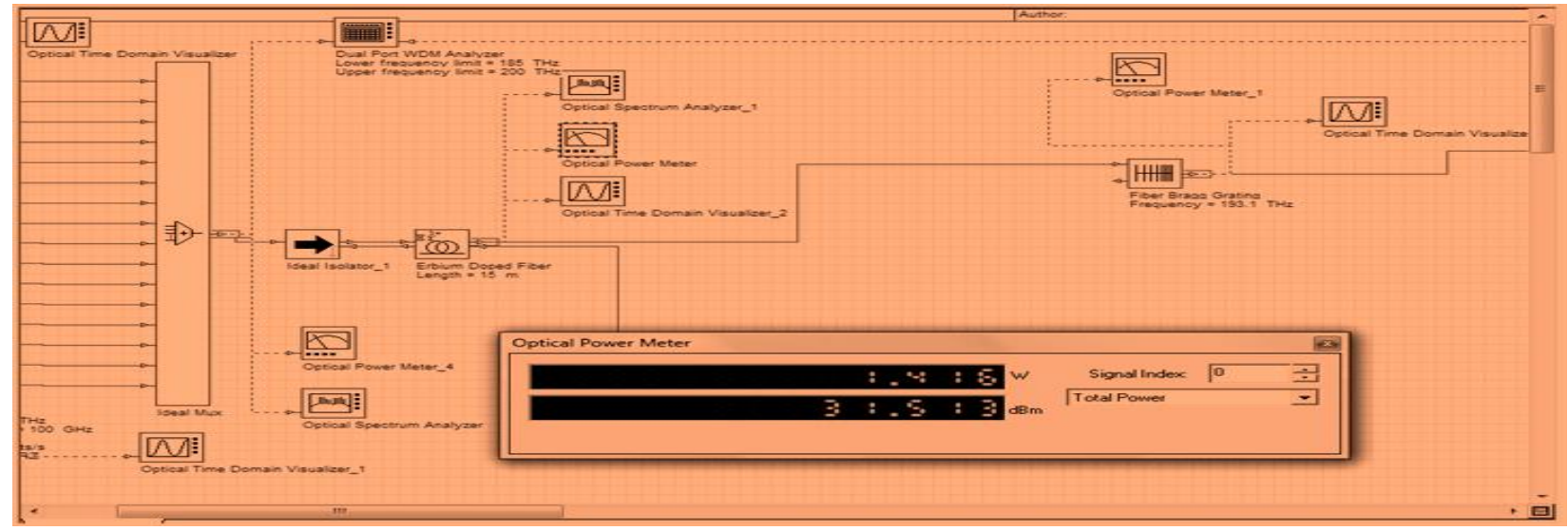

Fig. 6.1 optical power meter 4 reading

6.2 Consulidated Result of Single Stage EDFA

Table-6.1 Value of signals on each optical power meter at different distance for single stage EDFA

\begin{tabular}{|c|c|c|c|c|c|c|c|}
\hline S. No & Length & $\begin{array}{c}\text { Input } \\
\text { power } \\
(\mathrm{MW})\end{array}$ & $\begin{array}{c}\text { Received } \\
\text { power (MW) }\end{array}$ & Avg. Gain & Avg. Noise & $\begin{array}{c}\text { Input OSNR } \\
\text { (DB) }\end{array}$ & $\begin{array}{c}\text { Output OSNR } \\
\text { (DB) }\end{array}$ \\
\hline 1 & 5 & $8.71 \mathrm{MW}$ & $1.096 \mathrm{~W}$ & 17.9050 & 16.81 & 97.2398 & 38.362 \\
\hline 2 & 10 & $8.71 \mathrm{MW}$ & $0.871 \mathrm{~W}$ & 16.4488 & 17.3416 & 97.2398 & 37.8437 \\
\hline 3 & 15 & $8.71 \mathrm{MW}$ & $0.691 \mathrm{~W}$ & 15.0656 & 17.6629 & 97.2398 & 37.5232 \\
\hline 4 & 20 & $8.71 \mathrm{MW}$ & $0.549 \mathrm{~W}$ & 13.6859 & 18.0432 & 97.2398 & 37.1435 \\
\hline
\end{tabular}




\subsection{Multi Stage EDFA Results}

The Optisystem product is used to plan EDFA in the WDM Framework. The structure consists of 16 information signals (channels), a right multiplexer, four isolators, a pump laser, erbium-doped fiber with a length of $5 \mathrm{~m}$, demultiplexer, photo-localization pin, and a low-pass basel channel. , As shown in Fig. 1 das, that the scaffold consists of 16 multiplex movements, with a channel division of $1003 \mathrm{z}$ with an optimized wavelength in the wavelength range of 193.1 Thz. The energy of each channel is $0 \mathrm{dBm}$. Pumping at $980 \mathrm{~nm}$ is used to bring $0.75 \mathrm{~W}$ doped Iotas to a higher standard of living.

The isolator was copied from doped erbium fibers. The first isolator (Isolator_1) designed by EDF designs a laser with a repeat of $980 \mathrm{~nm}$ and a power of $0.75 \mathrm{~W}$.

If the correct multiplexer and the correct multiplexer signals are detected in the EDFA case with multiple arrangements, the signals are first estimated on the optical power meter (optical power meter_1). Figure 4 shows an estimate of the signals at the beginning of this operation.

\subsection{Consulidated Result Of Single Stage EDFA}

Table-6.2 Value of signals on each optical power meter at different distance for multistage EDFA

\begin{tabular}{|c|c|c|c|c|c|c|c|}
\hline $\begin{array}{c}\text { S. } \\
\text { No }\end{array}$ & Length & Input power(MW) & $\begin{array}{c}\text { Received } \\
\text { power (MW) }\end{array}$ & Avg. Gain & $\begin{array}{c}\text { Avg. } \\
\text { Noise }\end{array}$ & $\begin{array}{c}\text { Input OSNR } \\
\text { (DB) }\end{array}$ & $\begin{array}{c}\text { Output } \\
\text { OSNR (DB) }\end{array}$ \\
\hline 1 & 5 & $8.71 \mathrm{MW}$ & 1.099 & 17.9162 & 7.6104 & 97.2398 & 47.5911 \\
\hline 2 & 10 & $8.71 \mathrm{MW}$ & 0.873 & 16.3942 & 8.1370 & 97.2398 & 47.0691 \\
\hline 3 & 15 & $8.71 \mathrm{MW}$ & 0.693 & 15.0751 & 8.4620 & 97.2398 & 46.7500 \\
\hline 4 & 20 & $8.71 \mathrm{MW}$ & 0.551 & 13.6940 & 8.8504 & 97.2398 & 46.3689 \\
\hline
\end{tabular}

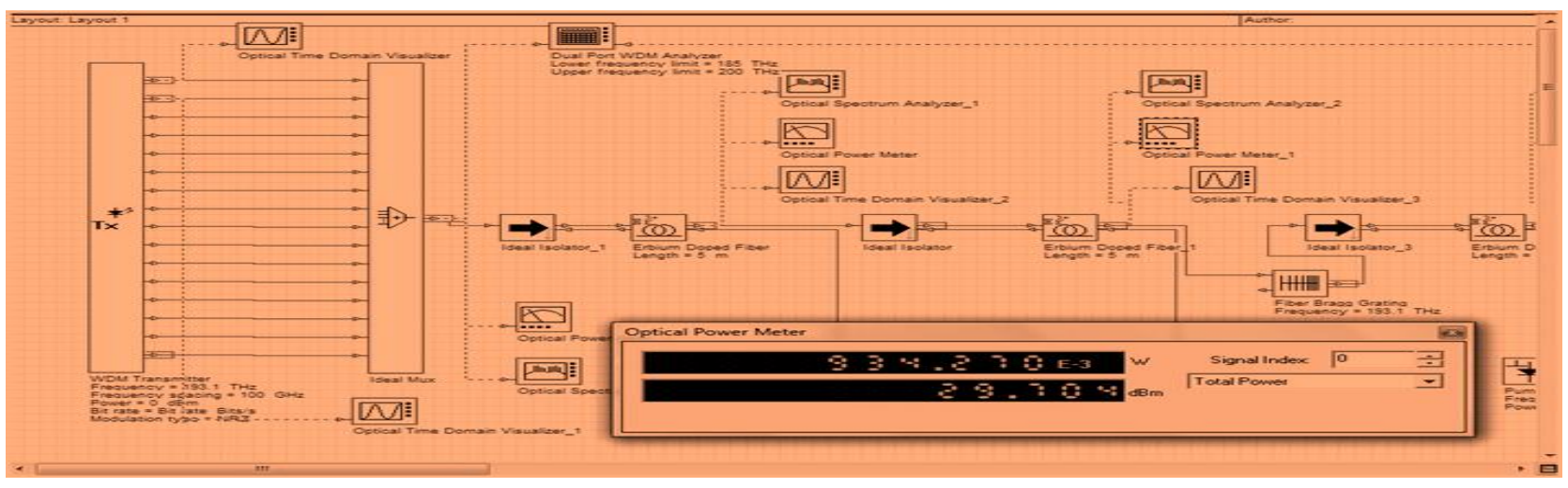

Fig. 6.2 Optical Power Meter_1 Reading

\section{CONCLUSION}

As shown above, the proposed system has been successfully implemented and tested in a startup optics environment. As guaranteed, the proposed design can provide higher signal-to-noise ratios and noise (dB) than single-phase EDFA with similar outputs. Optical performance has increased significantly compared to single-phase EDFA. The strategic positioning of EDFA at several stages before and after FBG, improves some power consumption. As shown, there is an average DB tool. The figure shows the noise and MW related to optical power, as can be seen from the result.

Table A Comparison between single stage and multi stage EDFA Results

\begin{tabular}{|c|c|c|c|c|c|c|c|c|c|c|}
\hline \multicolumn{4}{|c|}{} & \multicolumn{4}{c|}{ Single Stage EDFA Result } & \multicolumn{3}{c|}{ Multi Stage EDFA Result } \\
\hline S.No. & Length & $\begin{array}{c}\text { Input } \\
\text { power } \\
\text { (MW) }\end{array}$ & $\begin{array}{c}\text { Received } \\
\text { power } \\
\text { (MW }\end{array}$ & $\begin{array}{c}\text { Avg. } \\
\text { Gain }\end{array}$ & $\begin{array}{c}\text { Avg. } \\
\text { Noise } \\
\text { Figure }\end{array}$ & $\begin{array}{c}\text { Output } \\
\text { OSNR } \\
(\mathrm{DB})\end{array}$ & $\begin{array}{c}\text { Received } \\
\text { power } \\
\text { (MW) }\end{array}$ & $\begin{array}{c}\text { Avg. } \\
\text { Gain }\end{array}$ & $\begin{array}{c}\text { Avg. } \\
\text { Noise } \\
\text { Figure }\end{array}$ & $\begin{array}{c}\text { Output } \\
\text { OSNR } \\
\text { (DB) }\end{array}$ \\
\hline 1 & 5 & $\begin{array}{c}8.71 \\
\text { MW }\end{array}$ & $1.096 \mathrm{~W}$ & 17.9050 & 16.81 & 38.362 & 1.099 & 17.9162 & 7.6104 & 47.5911 \\
\hline 2 & 10 & $\begin{array}{c}8.71 \\
\text { MW }\end{array}$ & $0.871 \mathrm{~W}$ & 16.4488 & 17.3416 & 37.8437 & 0.873 & 16.3942 & 8.1370 & 47.0691 \\
\hline 3 & 15 & $\begin{array}{l}8.71 \\
\text { MW }\end{array}$ & $0.691 \mathrm{~W}$ & 15.0656 & 17.6629 & 37.5232 & 0.693 & 15.0751 & 8.4620 & 46.7500 \\
\hline 4 & 20 & $\begin{array}{c}8.71 \\
\text { MW }\end{array}$ & $0.549 \mathrm{~W}$ & 13.6859 & 18.0432 & 37.1435 & 0.551 & 13.6940 & 8.8504 & 46.3689 \\
\hline
\end{tabular}

After comparison, we found that the difference between single-level and multi-level results, multi-level retrieval performance and OSNR output is greater than single-level EDFA. Average Noise levels are lower than the individual 
levels of EDFA and media. Profits are small. Therefore, all multi-stage EDFA experiments provide an excellent result compared to single-stage EDFA.

\section{FUTURE SCOPE}

WDM systems are the lifeline of modern fiber optic networks. As our work suggests, the use of multi-stage EDFA is comparable to single-stage EDFA. EDFA can bring significant improvements in noise and optical performance. This means that if it is a research path, the performance of the WDM system can be significantly increased, and much more is needed to meet the increasing bandwidth requirement. Highly effective dispersion compensation can be obtained using five lattice fibers. In addition, ongoing research on EDFA provided an optical amplifier to use more sophisticated warfare components and components than EDFA. Another research focus is the application of nanotechnology to manufacture a low-loss optical amplifier. With this series of new techniques, many things will happen with this decoding. [12, 13, 15].

\section{REFERENCES}

[1] Tomas Ivaniga, LubosOvsenik, Jan Turan "The Four-Channel WDM System Using Semiconductor Optical Amplifier" IEEE 2016.

[2] Ch. Pranavi\& B. Geetha Rani "Uniform FBG as Dispersion Compensator in Optical Fiber Communications" IJIR 2017.

[3] Pargat Singh \& Dr. Charanjit Singh “A Carrier Generated Self Phase Modulation Reliant Rof System Employing RSOA As Modulator For Upstream RAUs And Incorporating DCF, FBG For Dispersion Compensation” IJARCS 2017.

[4] P. S. Rath, Ajeet Satyam, Diwakar Mani Jha, Iftekhar Khalid "Performance Analysis of Long Fiber Optic Link using Fiber Bragg Grating for Dispersion Compensation” IJESC 2017.

[5] Rupinder Kaur, Mandeep Singh "Analysis on Dispersion Compensation with DCF based on Optisystem-A Review" IJOES 2016.

[6] Sachindev, Col. (Dr.) Suresh Kumar "Dispersion Compensation in Optical Fiber Communication using Bragg Grating” ICRISTM 2016.

[7] SahilKakalia and Munish Singh "Performance Analysis of DWDM System Having 0.8- Tbps Date Rate with 80 Channels" IJST 2016.

[8] B.Geetha Rani \& Ch. Pranavi "Dispersion Compensation In OFC Using FBG” IJERGS 2016.

[9] R. K. Sethi\& Dr. Aditya Goel "Performance Analysis of Optical Communication Systems using OFDM by Employing QPSK Modulation” IJRITCC 2015.

[10] Jiangbing Du, Lu Li, Xinyu Fan, Qingwen Liu and Zuyuan He "Sensitivity Enhancement for Fiber Bragg Grating Sensors by Four Wave Mixing” SJTU 2015.

[11] Ranjita Rout \&Subhrajit Pradhan \&SrikantaPatnaik "Role of DCF technique for enhancing optical fiber communication System utility" IRJET 2015.

[12] Aashima Bhardwaj, Gaurav Soni "Performance Analysis of Optical Communication System Using Fiber Bragg Grating” SSRG-IJECE 2015.

[13] Manpreet Kaur, HimaliSarangal "Simulation of Optical Transmission System to Compensate Dispersion Using Chirped Fiber Bragg Grating (FBG)" IJARCCE 2015.

[14] Tarun Bansal \& Mr. P.S.Bhullar "Designing High Data Rate Long Haul Optical Communication System $96 \times 80$ $\mathrm{Gb} / \mathrm{s}$ transmission using hybrid Raman-Erbium doped optical amplifiers with Improved Performance using DWDM" IJAR 2015.

[15] MulayamYadav\& A. K. Jaiswal\&Neelesh Agrawal \&NavenduNitin "Design Performance of High Speed Optical Fiber WDM System with Optimally Placed DCF for Dispersion Compensation” IJCA 2015.

[16] Latika Chaudhary Pruthi1, SurajNagpal "Analysis of FBG Parameters of TANH Profile for Dispersion Compensation" IJARET 2015. 